\title{
Physical frailty, disability, and dynamics in health perceptions: a preliminary mediation model
}

\author{
This article was published in the following Dove Press journal: \\ Clinical Interventions in Aging \\ 2 March 2016 \\ Number of times this article has been viewed
}

\author{
Anna Mulasso \\ Mattia Roppolo \\ Emanuela Rabaglietti \\ Department of Psychology, University \\ of Torino, Torino, Italy
}

Purpose: Frailty is a condition characterized by loss of functional reserve and altered homeostatic capacity. The aging process is related with complex indicators of physiological state. This study aims, with a preliminary mediation model, to reveal the possible role of mediator of health perceptions variability in the relationship between frailty and disability.

Patients and methods: A longitudinal study (100 days) was performed. Data from 92 institutionalized older adults were used in the analysis. Frailty was assessed in baseline using the Italian version of the Survey of Health, Ageing and Retirement in Europe - Frailty Instrument; health perceptions were assessed on a daily basis by three visual analog scale questions; and disability was measured in baseline and post-test using the Katz Activities of Daily Living questionnaire. The product-of-coefficient mediation approach was used to test direct and indirect effects of frailty.

Results: Results showed that daily variability of health perceptions plays the role of mediator between frailty and disability. In all the steps, statistically significant results were found.

Conclusion: This preliminary result may indicate that physical frailty increases the variability in health perceptions contributing to disability.

Keywords: functional decline, loss of autonomy, variability, health outcomes, dynamic systems

\section{Introduction}

Frailty has been conceptualized as a loss of functional reserve, inability to react to life events, augmented risks for negative health outcomes, and altered homeostatic capacity caused by a multiple and interactive complex system. ${ }^{1-4}$ Gobbens et $\mathrm{al}^{5,6}$ defined frailty as "a dynamic state affecting an individual who experiences losses in one or more domains of human functioning." Definitions of frailty suggest that the decrease in functional ability is a dynamic and complex system, which may cause instability in the physiological status. In fact, previous studies demonstrated how the aging process and frailty status are connected with indicators of variability and complexity in the functional and physiological status. ${ }^{7,8}$ Furthermore, from dynamic systems theory, it is known that variability provides important information about the developmental process, giving meaningful information about the possible drastic changes, transition phases, bifurcations, and stable states. ${ }^{9}$ However, currently it is not known if frailty status may be connected with indicators of dynamics on a comprehensive and individual health assessment. For this reason, this preliminary study aims to understand if frailty status is related with daily variability on health perceptions and if this dynamic indicator plays a role as mediator in the relation between frailty status and disability.
Correspondence: Mattia Roppolo Department of Psychology, University of Torino, Via Verdi, I0, I0I24, Torino, Italy Tel +390 II 6702788

Fax +39 0116702791

Email mattia.roppolo@unito.it 


\section{Material and methods}

\section{Study population and procedures}

The study involved 116 institutionalized older adults, living in four residential care facilities in Italy. The following inclusion criteria were set: 1) living in residential care facilities, 2) older than 70 years, 3) Mini-Mental State Examination (MMSE) score higher than 25 (indicating absence of cognitive impairments). ${ }^{10}$ From the original sample ( $\mathrm{n}=116)$, data from 92 participants were used here. Twenty-four people were excluded because they did not complete the observational period (100 days) for reasons not related to study participation, or because they presented missing values in their data.

The Ethical Committee of the University of Torino approved the study. All participants provided written informed consent and they did not receive any incentives or reward for participating.

\section{Measures}

Frailty was assessed in baseline using the Italian version of the Survey of Health, Ageing and Retirement in Europe Frailty Instrument (SHARE-FI), consisting of five indicators: exhaustion, weight loss, weakness, slowness, and level of activity. ${ }^{11}$ SHARE-FI provides a continuous score ranging from 0 to 5 with an associated categorical label (nonfrail, prefrail, and frail). Higher scores correspond to a more serious frailty condition.

Health perceptions were measured on a daily basis (100 data points) with a specifically designed self-report instrument. The questionnaire is consistent with a previously developed conceptual model of health-related quality of life based on the dynamic systems approach, and was previously tested and used in an empirical study. ${ }^{12,13}$ The instrument was composed by three visual analog scale questions. The questions were: 1) How would you rate your physical health today?, 2) How would you rate your psychological/mental health today?, and 3) How satisfied are you about the social activities performed today? Data ranged from 0 (worst health possible or completely unsatisfied) to 1 (best health possible or completely satisfied). Health perceptions variability (HPV) was operationalized as (the average of) the absolute difference between two subsequent daily data points in each of the three items.

Disability was measured in baseline and post-test using the Katz Activities of Daily Living (ADL) questionnaire. ${ }^{14,15}$ This is a widely used instrument assessing six functions: bathing, dressing, going to toilet, transferring, feeding, and continence. The highest score is 6 and corresponds to absence of disability.

\section{Statistical analysis}

All the analyses were conducted with Statistical Package for Social Sciences (SPSS), version 21.0 (SPSS Inc, Chicago, IL, USA). Statistical significance level was fixed at 0.05 for all tests.

To test the relation among frailty status, HPV and disability a mediation model was performed, according to the product-of-coefficient approach. ${ }^{16}$ To perform the productof-coefficient mediation, the following steps were executed: 1) $\alpha$ coefficient of mediation, regressing the HPV scores on frailty status; 2) $\beta$ coefficient, regressing the post-test ADL onto frailty status, baseline ADL, and HPV; 3) $\alpha \beta$ coefficient, multiplying the two previously cited regression coefficients; 4) the $z$ scores associated with the mediated effect dividing the $\alpha \beta$ coefficient by its standard error. $\alpha$ and $\beta$ coefficients are, respectively, a test for the action and the conceptual theory, used in mediation analyses. Furthermore, $\tau^{\prime}$ coefficient, representing the unmediated intervention effects, was computed.

\section{Results}

\section{Participants characteristics}

The whole sample had a mean age of $84.3 \pm 5.9$ years. The majority of participants were women $(n=62 ; 67.4 \%)$. Most of the participants reported to have a comorbidity condition $(\mathrm{n}=70 ; 76.1 \%)$, and the mean number of reported diseases was 2.6 \pm 1.3 . The mean score of MMSE was 28.2 \pm 1.8 . Half of the participants ( $\mathrm{n}=46 ; 50.0 \%)$ were frail, while $20(21.7 \%)$ were prefrail and $26(28.3 \%)$ robust. The mean level of HPV was $0.09 \pm 0.04$. Finally, the baseline level of ADL was $4.5 \pm 1.1$, while the final score was $3.0 \pm 1.5$.

\section{Mediation}

\section{Action theory test}

The results of the action theory test are shown in Table 1 ( $\alpha$ coefficient). Results highlighted that frailty status caused a significant increase in the level of HPV $(P<0.001)$.

\section{Conceptual theory test}

The data presented in Table 1 ( $\beta$ coefficient) showed the significant relation between the HPV and ADL, confirming the conceptual theory test. In particular, it is possible to observe a negative association between the mediator and the outcome $(P<0.001)$.

\section{Mediated effects}

The model showed a statistically significant mediating effect (Table 1, $\alpha \beta$ coefficient). The data revealed negative mediated effects of HPV on ADL $(P<0.001)$, meaning 
Table I Single mediation model on disability

\begin{tabular}{lllllll}
\hline Mediator & $\alpha(\mathrm{SE})$ & $\beta(\mathrm{SE})$ & $\alpha \beta(\mathrm{SE})$ & $95 \% \mathrm{Cl}$ of $\alpha \beta$ & $\mathrm{z}$ & $\alpha \beta /\left(\alpha \beta+\tau^{\prime}\right)$ \\
\hline HPV & $0.022(0.004)^{*}$ & $-11.684(2.310)^{*}$ & $-0.257(0.005)^{*}$ & -0.406 to -0.134 & -51.40 & 0.181 \\
\hline
\end{tabular}

Notes: $* P<0.01 ; \alpha$ estimate of frailty status (unstandardized regression coefficient) on score of HPV; $\beta$ estimate of the independent effect of the mediator (unstandardized regression coefficient) on scores of ADL; $\alpha \beta$ product of coefficient estimate; mediated effect; $\mathrm{SE} ; 95 \% \mathrm{Cl}$ of $\alpha \beta$ 95\% confidence interval of the mediated effect; $z$ standard deviated associated with mediated effect (used for significance testing); $\alpha \beta /\left(\alpha \beta+\tau^{\prime}\right)$ proportion of mediated effect.

Abbreviations: ADL, Activities of Daily Living; Cl, confidence interval; HPV, health perceptions variability; SE, standard error.

that a higher rate of variability corresponded to a greater disability.

The mediated effect proportion was 0.18 , corresponding to a medium proportion of mediation. In all the steps, statistically significant results were found.

\section{Discussion}

These preliminary results demonstrated that frailty status, HPV, and disability were related to each other. Moreover, HPV plays a role as mediator in the relation between frailty status and disability.

Our results confirm previous findings about the dynamics and complexity of physiological indicators in frail individuals. ${ }^{7,8}$ Moreover, they confirm the hypothesis that frailty is connected to day-to-day fluctuations of health outcomes because of instability in the functional systems. ${ }^{17}$ Furthermore, data reported here are in line with the conceptualization of frailty as a dynamic state as proposed by Gobbens et al. ${ }^{6}$

This preliminary study provides more specific insights about the mechanisms of frailty in the relation with health outcomes. The direct effect of frailty on the health outcome is confirmed, as found in previous studies. ${ }^{17-23}$ However, as a further step, we found that this direct effect is mediated by fluctuations of health perceptions. On the one hand this finding is in line with the current theories of frailty, ${ }^{6,17}$ and on the other hand represents a first preliminary evidence about the mechanisms of action between frailty status and health outcomes. Our results suggest that it is important to focus on individual-based time-series analysis with the use of dynamics indicators of health and functioning, in order to better predict and understand the pathways between frailty and disability. Such an approach may lead to a better knowledge of health decline during the later life and to prevent negative health outcomes.

The main limitation of our study is related to the sample, because of its small size in comparison with demographic or clinical studies. However, the sample size and the number of observations collected for this paper are generally sufficient for the study of individual characteristics and development. Moreover, our study is a preliminary investigation. Further studies need to enroll a larger sample including also individuals with cognitive decline, in order to give a more precise picture of the relations between physical frailty, variability in health perceptions, and disability in institutionalized older adults.

\section{Conclusion}

As a conclusion, it is possible to hypothesize that physical frailty reduces the ability to adapt with stressors and react to life events, increasing the levels of HPV and contributing to a worsening of health outcomes in a sample of institutionalized older adults. This hypothesis needs to be confirmed with wider studies.

\section{Acknowledgments}

For the publication of this article funding has been received from the project "Sistema di allerta integrato delle fragilità emergenti" within the Regional call "Bando Regionale a sostegno di progetti di ricerca industriale e/o sviluppo sperimentale di applicazioni integrate e innovative in ambito Internet of Data" funded by Regione Piemonte and the "Fondo Europeo di Sviluppo Regionale (POR-FESR)." The funding bodies were not involved in the study design, data collection, and analysis or in writing the report.

\section{Disclosure}

Anna Mulasso and Mattia Roppolo received a research fellowship from the Department of Psychology, University of Torino (reference number 17/2015, protocol n. 320) funded by "Regione Piemonte" and the "Fondo Europeo di Sviluppo Regionale (POR-FESR)" for the project "Sistema di allerta integrato delle fragilità emergenti." The authors report no other conflicts of interest in this work.

\section{References}

1. Fried LP, Tangen CM, Walston J, et al. Frailty in older adults evidence for a phenotype. J Gerontol A Biol Sci Med Sci. 2001;56(3):M146-M157.

2. Fried LP, Ferrucci L, Darer J, Williamson JD, Anderson G. Untangling the concepts of disability, frailty, and comorbidity: implications for improved targeting and care. J Gerontol A Biol Sci Med Sci. 2004;59(3): M255-M263.

3. Hubbard RE, Rockwood K. Frailty in older women. Maturitas. 2011; 69(3):203-207.

4. Rockwood K, Hogan DB, MacKnight C. Conceptualisation and measurement of frailty in elderly people. Drugs Aging. 2000;17(4):295-302. 
5. Gobbens RJJ, Luijkx KG, Wijnen-Sponselee MT, Schols JMGA. In search of an integral conceptual definition of frailty: opinions of experts. J Am Med Dir Assoc. 2010;11(5):338-343.

6. Gobbens RJ, Luijkx KG, Wijnen-Sponselee MT, Schols JM. Towards an integral conceptual model of frailty. J Nutr Health Aging. 2010; 14(3):175-181.

7. Lipsitz LA. Physiological complexity, aging, and the path to frailty. Sci Aging Knowl Environ. 2004;2004(16):pe16.

8. Montero-Odasso M, Muir SW, Hall M, et al. Gait variability is associated with frailty in community-dwelling older adults. J Gerontol A Biol Sci Med Sci. 2011;66A(5):568-576.

9. Kunnen SE. A Dynamic Systems Approach of Adolescent Development. London: Psychology Press; 2012.

10. Folstein MF, Folstein SE, McHugh PR. "Mini-mental state": A practical method for grading the cognitive state of patients for the clinician. $J$ Psychiatr Res. 1975;12(3):189-198.

11. Romero-Ortuno R, O'Shea D, Kenny RA. The SHARE frailty instrument for primary care predicts incident disability in a European population-based sample. Qual Prim Care. 2011;19(5):301-309.

12. Roppolo M, Kunnen SE, Mulasso A, Van Geert P, Rabaglietti E. Older adults and Health Related Quality of Life: a conceptual model based on dynamic systems. In: Wolfe R, editor. Psychological Health and Needs Research Developments. I. Psychology. Hauppauge, NY: Nova Science Publishers; 2015:131-156.

13. Roppolo M. How Do You Feel Today?: The Use of a Dynamic Systems Approach in the Conceptualization and Analysis of Health Related Quality of Life in Older Adults. Groningen: University of Groningen; 2015. ISBN: 9789036782388.

14. Katz S, Downs TD, Cash HR, Grotz RC. Progress in development of the index of ADL. Gerontologist. 1970;10(1 Part 1):20-30.
15. Wallace M, Shelkey M. Katz Index of Independence in Activities of Daily Living (ADL). Urol Nurs. 2007;27(1):93-94.

16. Cerin E, MacKinnon DP. A commentary on current practice in mediating variable analyses in behavioural nutrition and physical activity. Public Health Nutr. 2009;12(08):1182.

17. Clegg A, Young J, Iliffe S, Rikkert MO, Rockwood K. Frailty in elderly people. Lancet Lond Engl. 2013;381(9868):752-762.

18. Roppolo M, Mulasso A, Gobbens R, Mosso C, Rabaglietti E. A comparison between uni- and multidimensional frailty measures: prevalence, functional status, and relationships with disability. Clin Interv Aging. 2015;10:1669-1678.

19. Ensrud KE, Ewing SK, Cawthon PM, et al. A comparison of frailty indexes for the prediction of falls, disability, fractures, and mortality in older men. J Am Geriatr Soc. 2009;57(3):492-498.

20. Ensrud KE, Ewing SK, Taylor BC, et al. Comparison of 2 frailty indexes for prediction of falls, disability, fractures, and death in older women. Arch Intern Med. 2008;168(4):382-389.

21. Hoogendijk EO, van der Horst HE, Deeg DJH, et al. The identification of frail older adults in primary care: comparing the accuracy of five simple instruments. Age Ageing. 2013;42(2):262-265.

22. Woo J, Leung J, Morley JE. Comparison of frailty indicators based on clinical phenotype and the multiple deficit approach in predicting mortality and physical limitation. J Am Geriatr Soc. 2012;60(8):1478-1486.

23. Mulasso A, Roppolo M, Rabaglietti E. The role of individual characteristics and physical frailty on health related quality of life (HRQOL): A cross sectional study of Italian community-dwelling older adults. Arch Gerontol Geriatr. 2014;59(3):542-548.
Clinical Interventions in Aging

\section{Publish your work in this journal}

Clinical Interventions in Aging is an international, peer-reviewed journal focusing on evidence-based reports on the value or lack thereof of treatments intended to prevent or delay the onset of maladaptive correlates of aging in human beings. This journal is indexed on PubMed Central, MedLine,

\section{Dovepress}

CAS, Scopus and the Elsevier Bibliographic databases. The manuscript management system is completely online and includes a very quick and fair peer-review system, which is all easy to use. Visit http://www.dovepress. com/testimonials.php to read real quotes from published authors. 